\title{
METAMORPHOSE VI - the Virtual Institute for artificial electromagnetic materials and metamaterials: origin, mission, and activities
}

\author{
Filiberto Bilotti ${ }^{1, *}$, Carsten Rockstuhl ${ }^{2}$, Alex Schuchinsky ${ }^{3}$, and Sergei Tretyakov ${ }^{4}$ \\ 1 Roma Tre University, Department of Engineering, Rome 00146, Italy \\ 2 Karlsruhe Institute of Technology, Institute of Theoretical Solid State Physics, Karlsruhe 76128, Germany \\ 3 Queen's University of Belfast, Institute of Electronics, Communications and Information Technology, Belfast BT7 1NN, UK \\ 4 Aalto University, School of Electrical Engineering, Aalto 00076, Finland
}

Received 15 February 2014 / Accepted 7 April 2014

\begin{abstract}
In this paper, we outline the background, mission, and activities of the Virtual Institute for Artificial Electromagnetic Materials and Metamaterials (METAMORPHOSE VI). This international association, founded in the framework of the FP-6 Network of Excellence METAMORPHOSE, aims at promoting and developing research, training, and dissemination activities in the emerging and highly dynamic field of advanced electromagnetic materials and metamaterials at both European and International levels. More than 300 researchers are currently associated with the METAMORPHOSE VI which networks them together in a learnt society. After a brief description of the association and its mission, we present an overview of the activities developed by the METAMORPHOSE VI, with a particular emphasis on the coordination of the European Doctoral Program on Metamaterials (EUPROMETA) and the organization of the International Congress on Advanced Electromagnetic Materials in Microwaves and Optics - metamaterials congress.
\end{abstract}

Key words: Metamaterials, Education, Dissemination, Metamaterials Congress.

\section{Origin of the METAMORPHOSE VI}

The Virtual Institute for Artificial Electromagnetic Materials and Metamaterials (METAMORPHOSE VI) [1] is a not-forprofit international association, whose purposes include research, education, and promotion of artificial electromagnetic materials and metamaterials. The association, established in Belgium in 2007, has been founded by the partners of the Network of Excellence (NoE) "Metamaterials organized for radio, millimeter wave, and photonic superlattice engineering" METAMORPHOSE funded by the European Commission within the 6th Framework Program (2004-2008). This NoE has been established within the Priority 3: nano-technologies and nano-sciences, knowledge-based multifunctional materials, and new production processes and devices; Research theme 3.4.2: knowledge-based multifunctional materials development of fundamental knowledge, understanding materials phenomena of the activity area nano-technologies and nano-sciences, knowledge-based multifunctional materials, and new production processes and devices (NMP).

*e-mail: bilotti@uniroma3.it
The NoE, coordinated by Prof. Sergei Tretyakov (Aalto University, former Helsinki University of Technology), started its activities in June 2004, lasted 4 years, and linked 24 academic and industrial partners from 13 European countries, see the partner list in Figure 1.

The main aim of NoE METAMORPHOSE was to coordinate and integrate the research efforts of the key players in the field of metamaterials in Europe, by reducing replication and fragmentation, and developing a new platform to share numerical and experimental research facilities among the partners. In order to make a lasting impact within the European research framework and industry, the NoE pursued the following medium- and long-term goals [2]:

(a) create an association which would continue the coordination, integration, training, and dissemination activities initiated by the NoE;

(b) establish a new highly reputable annual conference on metamaterials in Europe;

(c) develop and operate a training program for educating new generations of researchers and practitioners in the field;

(d) create a framework for scientific interactions, as a base for future European research. 


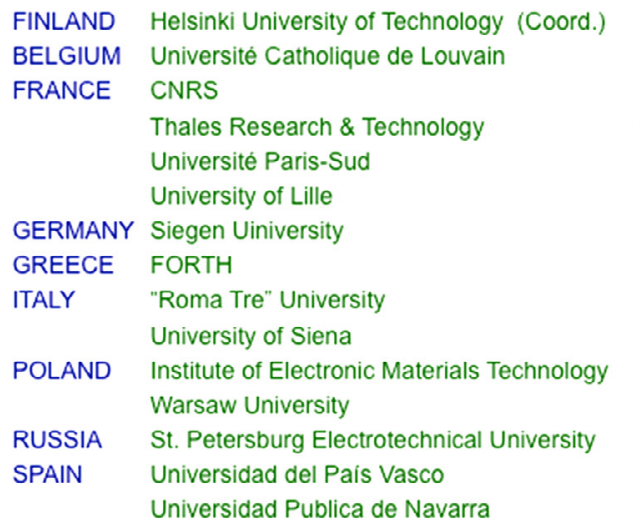

$\begin{array}{ll} & \text { Universitat Politechnica de Catalunya } \\ & \text { Universidad Autonoma de Barcelona } \\ \text { SWEDEN } & \text { Chalmers University } \\ \text { SWITZ. } & \text { Swiss Federal Inst. of Technology-EPFL } \\ \text { TURKEY } & \text { Bilkent University } \\ \text { UK } & \text { Queen's University of Belfast } \\ & \text { University of Glasgow, } \\ & \text { Loughborough University } \\ & \text { University of Southampton } \\ \text { START: } & \text { June 2004 } \\ \text { DURATION: } & 4 \text { years } \\ \text { INSTITUTIONS: } & 23 \\ \text { COUNTRIES: } & 13 \\ \text { BUDGET: } & 4.4 \text { MEuro }\end{array}$

Figure 1. List of the partners and key-facts of the FP-6 Network of Excellence METAMORPHOSE (2004-2008).

$\begin{array}{ll}\text { AALTO } & \text { Aalto University } \\ \text { UCL } & \text { Université Catholique de Louvain } \\ \text { UOS } & \text { University of Southampton } \\ \text { UniTN } & \text { University of Trento } \\ \text { UPNA } & \text { Universidad Publica de Navarra } \\ \text { UGLAS } & \text { University of Glasgow } \\ \text { ETU } & \text { St. Petersburg Electrotechnical University } \\ \text { FORTH } & \text { Foundation for Research and Technology } \\ \text { WU } & \text { University of Warsaw } \\ \text { Roma Tre } & \text { "Roma Tre" University } \\ \text { LOUGH } & \text { Lougborough University } \\ \text { UNISI } & \text { Univesità degli Studi di Siena } \\ \text { QUB } & \text { Queens University of Belfast } \\ \text { UPS } & \text { Université Paris-Sud } \\ \text { UAB } & \text { Universitat Autonoma de Barcelona } \\ \text { ITME } & \text { Institute of Electronic Materials Technology } \\ \text { SIEGEN } & \text { Universität Siegen } \\ \text { FSU } & \text { Friedrich-Schiller-Universität Jena } \\ \text { QMUL } & \text { Queen Mary University of London } \\ \text { GEA } & \text { University of Paris Ouest } \\ \text { DLR } & \text { German Aerospace Centre } \\ \text { ENEUR } & \text { "Sapienza" University of Rome } \\ \text { DIE } & \text { "Sapienza" University of Rome }\end{array}$

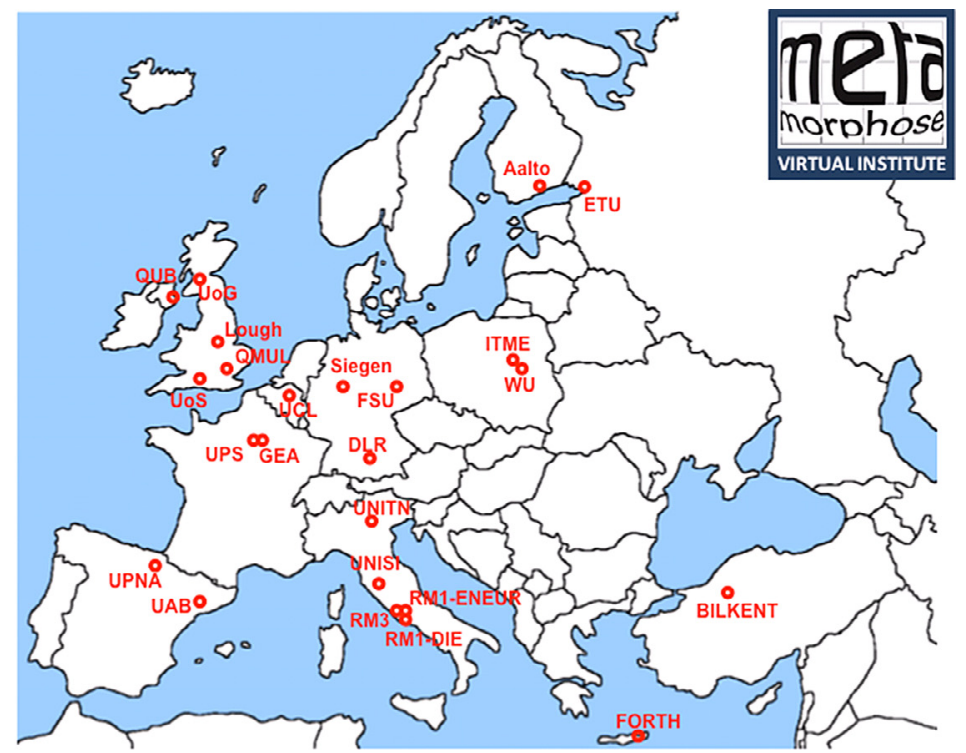

Figure 2. List and geographical distribution of the institutional members of the METAMORPHOSE VI.

\section{Mission of the METAMORPHOSE VI}

Following the NoE strategy, the METAMORPHOSE VI was registered in Belgium as a not-for-profit international association on May 5, 2007. In the very beginning the association has been founded by the two NoE partners, Helsinki University of Technology and "Roma Tre" University, with other 21 academic institutions joining it shortly. The association elected Prof. Sergei Tretyakov as its first President and Dr. Vladimir Podlozny as the first Secretary General, and established its operational office in Helsinki at Aalto University.

Nowadays, the METAMORPHOSE VI unites 23 institutional members from 12 European countries (see Figure 2 for the complete list and the geographic distribution) and connects together more than 300 professors, researchers, and students in an active research and education framework.
The expertise of the institutional members constituting the METAMORPHOSE VI encompasses phenomenology and theory, analytical and numerical modeling and simulations, fabrication of advanced electromagnetic materials, bottom-up and top-down nanofabrication of metamaterials, microwave and optical characterization of artificial electromagnetic materials and metamaterials, applications of artificial electromagnetic materials and metamaterials at microwave-THz-optical frequencies. Since 2012 the METAMORPHOSE VI has opened individual membership thus further broadening the portfolio of the association expertise.

The main mission of the METAMORPHOSE VI is to provide a credible platform for an international collaboration and interaction in the area of metamaterials and artificial electromagnetic materials. The association helps joining forces with colleagues and offers support and common voice within international and national organizations. 
In more detail, the mission of the METAMORPHOSE VI by constitution, consists in:

- integrating, managing, coordinating, and monitoring research projects in the field of artificial electromagnetic materials and metamaterials;

- spreading excellence within the scientific community and beyond, in particular, by organizing conferences and creating specialized journals in this field;

- creating and managing research programs in the field of metamaterials;

- activating and managing training programs, including training programs for students and industrial partners;

- providing information on artificial electromagnetic materials and metamaterials;

- transferring new technology in the field to the European industry;

- offering advice and services related to artificial electromagnetic materials and metamaterials to industries, producers, distributors, potential users, service suppliers, and to the like in Europe and worldwide.

\section{Main activities developed by the METAMORPHOSE VI}

In accordance with its mission, the METAMORPHOSE VI and its members develop institutional activities fostering the dissemination of the latest research results in the field of metamaterials and the training of doctoral students and practitioners working in industries and small-medium enterprises. More details of the three principal association activities are outlined below.

\subsection{Metamaterials congress}

The prime event held annually by the METAMORPHOSE VI is the metamaterials congress, founded originally by the METAMORPHOSE NoE. During its life, the NoE supported two main scientific meetings in the field of artificial electromagnetic materials: the Bianisotropics Conference, whose first edition dated back to 1993, and the Rome Workshop on Metamaterials and Artificial Materials for Electromagnetic Applications and TLC [3], which first held in 2003. In accordance with the NoE aim to reduce fragmentation, METAMORPHOSE members decided to amalgamate these two meetings in a single event, where the researchers from the two communities could get together and discuss the latest research results in the field of metamaterials. This decision has materialized in establishing the International Congress on Advanced Electromagnetic Materials for Microwaves and Optics - in short the metamaterials congress. Its first edition was held in Rome in October 2007. Since then it is organized on annual basis by the METAMORPHOSE VI jointly with an institution (not necessarily belonging to the association) which hosts the meeting and acts as a local organizer. To date, seven editions of the congress took place in Rome (2007), Pamplona (2008), London
(2009), Karlsruhe (2010), Barcelona (2011), St. Petersburg (2012), and Bordeaux (2013). The eighth edition of the congress will be held in Copenhagen in August 2014.

The congress represents a unique multidisciplinary forum where researchers from several diverse research communities meet and discuss the latest developments in field of artificial materials and metamaterials. The events usually attract 350 400 attendees and comprise a 4-day conference and a 2-day school on hot topics in the field. The conference format includes plenary talks on interdisciplinary topics, a few special sessions on the hot topics introduced by eminent keynote speakers, several invited talks given by recognized experts, contributed oral and poster presentations. In order to maintain the high quality of the conference program, all the papers are reviewed by at least two independent reviewers and are thoroughly checked against the originality and novelty criteria. Moreover, the reviewers are requested to give suggestions for improvements and further developments, which can be taken into account in updated final versions of paper abstracts. Since 2013, the congress proceedings are published in the IEEE Xplore electronic archive.

To encourage student participation in the congress, the organizers run student paper competition and a course of the European Doctoral Program on Metamaterials. Following the tradition of the workshops from which the metamaterials congress grew up, the METAMORPHOSE VI traditionally offers financial support to a few students and researchers from lowincome countries.

\subsection{European Doctoral Program on Metamaterials - EUPROMETA}

In order to educate new generations of researchers working actively in the field, the METAMORPHOSE NoE pioneered the European Doctoral Program on Metamaterials - EUPROMETA [4]. It was established in April 2005 and was led at that time by Prof. Filippo Capolino. This program, which is now a part of the METAMORPHOSE VI training activities, is managed by a consortium of 25 European academic institutions undersigned a Memorandum of Understanding which regulates the mutual recognition of credits (ECTS) among the partner institutions and defines the criteria for the students to receive the certificate Mention of Excellence in Metamaterials. To qualify for this certificate, students are to meet the stringent requirements:

- earn 5 ECTS upon attendance of EUPROMETA schools;

- successfully defend a $\mathrm{PhD}$ thesis on the topic directly related to metamaterials;

- visit at least one partner institution different from the home institution for three months;

- publish at least one journal article on metamaterials jointly with researchers not belonging to their own institutions, and have at least one further journal publication and three conference papers on metamaterials. The latter, are naturally linked to the participation in the Metamaterial Congress but that is, of course, not mandatory. 


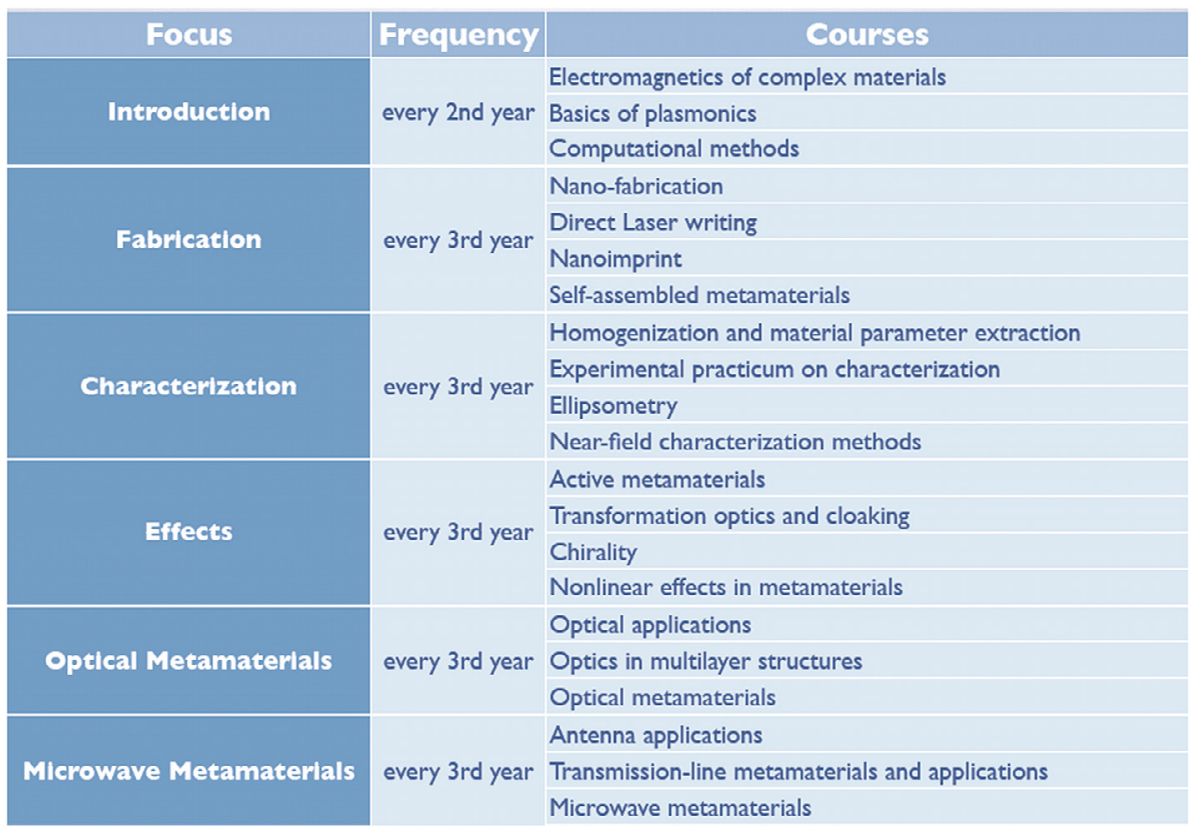

Figure 3. Program of the core courses offered by EUPROMETA.

As can be easily seen from this list of requirements, the overarching idea for the Mention of Excellence is to foster the collaboration among researchers from various institutions starting at the earlier stage and to facilitate their integration into an entire community.

The EUPROMETA educational activities are structured as a series of Distributed Doctoral School on Metamaterials and specialized courses covering the broad field of artificial electromagnetic materials and metamaterials. So far, EUPROMETA has organized 24 school events from two days to one week long each with an average of 20-40 attendees. The schools usually comprise a set of lectures, given by renowned experts in the field of metamaterials, exercises to deepen the understanding especially for some of the more advanced subjects, hands-on training in experimental techniques whenever this is appropriate, and visits to local laboratories. The latter promotes work and expertise of the local hosts to the school attendees. Also, a cultural program is an integral part of the school.

These schools, of course, serve primarily the educational purposes, but they also provide an excellent opportunity to bring together young and bright students who work on similar subjects albeit from different perspectives. Providing a creative and stimulating atmosphere is a key ingredient for incepting new ideas on how to further advance the field of metamaterials and how possible collaborations may look like. Since many of these students participate in the school events on a regular basis, long lasting exchange can be achieved and collaborative research subjects that eventually lead to publications can be developed very effectively.
The consortium organizes 2-3 schools every year and one of them is collocated with the metamaterials congress. The core courses of the Distributed Doctoral School on Metamaterials run every second or third year, according to the schedule presented in Figure 3 [5]. More specialized courses are also offered to expose students to the hot topics in the field.

Apart from the doctoral training, EUPROMETA delivers also some specialized courses for industries and small-medium enterprises.

\subsection{Research roadmap}

Since its outset, the METAMORPHOSE VI has been a driving force in the coordination and integration of research effort in the field of metamaterials. The key European researchers active in this field have been involved in the METAMORPHOSE VI. Their vision of the strategic research directions correlated with the future technological needs and capabilities, potential advances, and emerging applications are crystallized in the Research Road Map (RRM). This RRM differs from the conventional "roadmap" - it does not set priorities and define timelines. Its purpose is to present the METAMORPHOSE VI foresight of the exciting scientific challenges and new opportunities in exploration and exploitation of artificial electromagnetic materials and metamaterials. The RRM is structured at several levels, starting from the Grand challenges and application areas at the top. Then the strategic research targets and science and technology objectives, both science and 
application driven, are specified to identify the standing science and technology challenges within the envisioned impact areas.

To facilitate collaboration and expedite joint research efforts of the institutional members of the METAMORPHOSE VI, they meet every year at their research Workshop. Here they review and update the RRM to align it with the modern trends and state-of-the-art, and discuss future developments in the field of artificial electromagnetic materials and metamaterials. The latest version of the RRM is published at the METAMORPHOSE VI website [1].

\section{Conclusions}

In this paper, we have outlined the background, mission, and main activities of the METAMORPHOSE VI, the international association for promoting the research and education in artificial electromagnetic materials and metamaterials. The challenging mission of this non-profit organization is pursued through a structured platform for research interaction, education, and dissemination. The core activities developed in this framework, including the annual conference (metamaterials congress), the doctoral programs (EUPROMETA), and the association research roadmap have been presented in more detail. The interested reader can find more information at the websites of METAMORPHOSE VI http://metamorphose-vi.org and EUPROMETA http://school.metamorphose-vi.org, and in regular Newsletters published by the association operational office (http://www.metamorphose-vi.org/index.php/news).

\section{References}

1. http://metamorphose-vi.org.

2. F. Martín, F. Bilotti, I. Vendik, V. Podlozny, S. Tretyakov, A vision of metamaterials in Europe: the Network of Excellence METAMORPHOSE, Proceedings of the European Microwave Association 2 (2006) 101-106.

3. L. Vegni, A. Toscano, F. Bilotti, Rome 2006: Third Workshop on Metamaterials and Special Materials for Electromagnetic Applications and TLC, IEEE Antennas Propag. Mag. 48 (2006) 130-132.

4. F. Capolino, S. Tretyakov, F. Bilotti, A. Schuchinsky, F. Martin, V. Podlozny, A. Sihvola, D.A. Pawlak, I. Vendik, S. Zouhdi, C. Craeye, N. Johnson, J.M. Arnold, T. Szoplik, R. Gonzalo, METAMORPHOSE European Doctoral Programs on Metamaterials state-of-the-art, IEEE Antennas Propag. Mag. 48 (2006) 219-223.

5. C. Rockstuhl, A. Schuchinsky, F. Bilotti, V. Podlozny, S. Tretyakov, F. Capolino, Educating students on metamaterials - The story of the EUPROMETA, Proceedings of the 42nd European Microwave Conference (EuMC), Amsterdam, The Netherlands, 2012, pp. 491-493.

Cite this article as: Bilotti F, Rockstuhl C, Schuchinsky A \& Tretyakov S: METAMORPHOSE VI - the Virtual Institute for artificial electromagnetic materials and metamaterials: origin, mission, and activities. EPJ Appl. Metamat. $2014,1,1$. 